\title{
SYMPATHETIC OPHTHALMIA: VISUAL RESULTS WITH MODERN IMMUNOSUPPRESSIVE THERAPY
}

\author{
K. N. HAKIN, R. V. PEARSON, S. L. LIGHTMAN \\ London
}

\begin{abstract}
SUMMARY
Sympathetic ophthalmia is a rare bilateral panuveitis that follows penetrating injury to one eye. The use of systemic corticosteroids has transformed the prognosis, and good acuity in the sympathising eye can now be achieved. The use of immunosuppressive drugs, such as cyclosporin and azathioprine, in combination with the steroids, allows control of the intraocular inflammation at a much lower steroid dose, with concomitant reduction in the systemic side effects that accompany the use of systemic steroids.
\end{abstract}

One hundred and fifty years ago William McKenzie described sympathetic ophthalmia as one of the most dangerous inflammations to which the eye is exposed, stating that the most active treatment is ineffective and that renewed attacks generally result in total visual loss. The histopathology was reported in 1905 by Fuchs, who described a massive infiltration of the uvea by round cells with a preponderance of epithelioid cells and some giant cells and a tendency towards the formation of nodular aggregates on the inner surface of the choroid. ${ }^{1}$

Sympathetic ophthalmia is a clinical diagnosis based on the development of a bilateral panuveitis (when both eyes are present) following penetrating injury, and rarely intraocular surgery, to one eye. Studies have suggested an incidence of about $0.2 \%$ following accidental perforation and $0.01 \%$ following vitrectomy. ${ }^{2,3}$ Approximately $75 \%$ of cases develop within 3 months of insult although intervals as long as 42 years have been reported. ${ }^{4,5}$

Traditionally, the injured eye has been removed at the time of injury, or soon after, in an attempt to prevent the development of sympathetic ophthalmia, although there is no proof that this is actually of value. It is also unclear as to whether enucleation of the exciting eye confers any benefit once disease has started in the sympathising eye. Some feel that enucleation at this stage is valueless and should not be performed because the exciting eye may eventually have the better vision, or because the diagnosis may be

From: Moorfields Eye Hospital, London.

Correspondence to: Prof. S. L. Lightman, Moorfields Eye Hospital, City Road, London EC1V 2PD, UK. incorrect. $^{6,7}$ Others state that enucleation within 2 weeks of onset of sympathetic inflammation is associated with a relatively benign clinical course, and improves the visual outcome. ${ }^{4,8}$

There is no doubt, though, that medical therapy is of great value in this condition. The visual prognosis for established sympathetic ophthalmia was originally very poor, with only approximately $50 \%$ of cases retaining useful vision. ${ }^{7}$ The use of systemic corticosteroids and, more recently, immunosuppressive drugs such as azathioprine and cyclosporin has transformed the outlook of this potentially blinding disorder.

\section{PATIENTS AND RESULTS}

We have identified 18 patients with a diagnosis of sympathetic ophthalmia (Table I). Age at diagnosis ranged from 2.5 to 79 years, with follow-up from 7 months to 36 years. Fifteen cases followed trauma, 11 of which were after a known penetrating injury, and four after blunt trauma; at surgery, the latter were subsequently found to have posterior scleral rupture. Three cases were not associated with trauma but followed retinal detachment surgery, the exciting eye having had, on average, three operations prior to development of sympathetic ophthalmia. The interval between injury or initial surgery and diagnosis ranged from 1 month to 34 years with a mean of 70 months, with eight cases developing sympathetic ophthalmia within 1 year of insult. Five patients underwent enucleation of the exciting eye after the diagnosis was made; only one eye was enucleated prior to the onset of disease.

All patients developed anterior and posterior segment inflammation. The classical Dalen-Fuchs nodules were seen in 13 patients. All patients received topical medication, and all but one received systemic prednisolone, usually at a starting dose of $60 \mathrm{mg} /$ day that was tailed off as the inflammation subsided. Six patients required supplementary orbital floor injections of depot steroids, and nine patients received, in addition to the systemic steroids, azathioprine and/or cyclosporin A, either because of steroid intolerance or because of lack of response to 
Table I. Clinical details of 18 patients with sympathetic ophthalmia

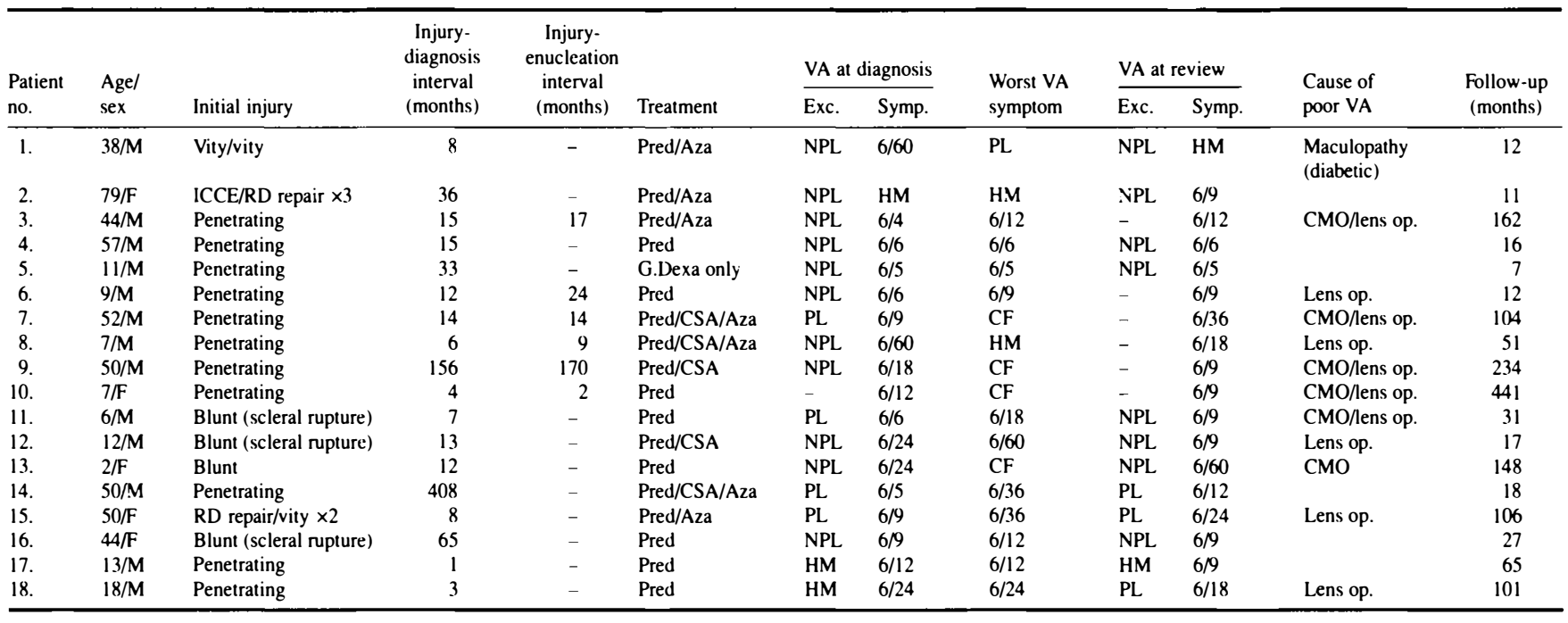

Abbreviations: Vity, vitrectomy; ICCE, intracapsular cataract extraction; RD, retinal detachment; Pred, prednisolone; Aza, Azathioprine; G. Dexa, guttae dexamethasone; CSA, cyclosporin A: VA, visual acuity; Exc., exciting; Symp., sympathising; NPL, no perception of light; PL, perception of light; HM, hand movement; CF, counting fingers; CMO, cystoid macular oedema; lens op., lens extraction.

steroid therapy alone. Slow withdrawal of treatment was attempted once inflammatory control had been established. All but two cases, however, relapsed at some time during follow-up and required reintroduction of or increase in therapy. Six patients suffered more than six relapses during the period of follow-up.

With their sympathising eye, 10 of 18 patients saw 6/9 or greater at review; only one saw less than $6 / 60$ but this patient did have pre-existing diabetic maculopathy. Poor visual acuity was due to macular oedema and/or lens opacities; seven patients have undergone lens extraction during the period of review. Six patients have required treatment for raised intraocular pressure in the sympathising eye during follow-up but only two are still on therapy. Apart from one eye which could perceive hand movements, all the exciting eyes that were not removed had either no perception of light or perception of light only.

At review, 11 patients are still receiving systemic steroid, although only one is on a dose of less than $10 \mathrm{mg} /$ day. There have been complications with treatment: with systemic steroids there has been deterioration of diabetic control, hypertension, myopathy, vertebral collapse and peptic ulceration; with cyclosporin A there has been raised creatinine and hypertrichosis.

\section{DISCUSSION}

Histopathological examination of an eye with sympathetic ophthalmia demonstrates a diffuse non-necrotising granulomatous inflammation. ${ }^{9}$ The choroid is thickened and contains a diffuse lymphocytic infiltrate, chiefly of T cells, with nests of epithelioid and giant cells. Dalen-Fuchs nodules, which are aggregates of retinal pigment epithelial (RPE), epithelioid and lymphocytic cells and which lie on the inner choroid beneath the RPE, are characteristic of sympathetic ophthalmia but are neither pathognomonic nor present in all cases.
The pathological appearance is generally thought to be the consequence of delayed hypersensitivity, but the antigens are unknown. Elschnig postulated in 1911 that injury to the exciting eye resulted in absorption and dissemination of the uveal pigment which produces the hypersensitivity reaction, initially in the injured eye and later in the sensitised tissue of the sympathising eye. ${ }^{10}$ There is no evidence, however, to support the idea that melanin is antigenic. An animal model of uveitis, experimental autoimmune uveoretinitis (EAU), can be induced by immunisation with a retinal soluble protein, retinal-S antigen." A diffuse granulomatous panuveitis is produced, which resembles some features of sympathetic ophthalmia but differs in the involvement of both the retina and choriocapillaris. ${ }^{12}$ The immune reaction alternatively may be directed towards surface membrane antigens that may be shared by photoreceptors, RPE cells and choroidal melanocytes. ${ }^{9}$ An infectious agent has also been postulated, either in isolation or in association with intrinsic antigen. ${ }^{13}$ A significantly high incidence of HLA-A11 has been found in one study, suggesting a possible genetic factor. ${ }^{8}$

It has been suggested that as the choroid has no lympatics, removal of any intraocular antigen by the blood could allow immunological tolerance. Any penetrating injury allows access of the intraocular antigen to the conjunctival lymphatics and then to the regional lymph nodes, which subsequently induces an immunological reaction towards the previously tolerated antigen. ${ }^{12}$

Once sympathetic inflammation is diagnosed, medical treatment should be initiated if vision is threatened or deteriorates and should follow that of any uveitis, i.e. topical steroids and mydriatics for anterior uveitis, systemic corticosteroids for posterior segment inflammation that threatens or reduces vision. An eye with posterior uveitis and an acuity of $6 / 6$ does not necessarily require treatment. If systemic therapy is required, we have used oral prednisolone, starting at around $60 \mathrm{mg} /$ day for 1 week and then slowly reducing the dose at weekly intervals, assum- 
ing inflammatory control has been achieved. There have been several published series which have demonstrated that systemic corticosteroids can confer a relatively benign clinical course, and improve visual prognosis. ${ }^{4,8,14}$ There are frequent complications, however, with the level and duration of steroid therapy that may be required to control inflammation. Glaucoma, which can be very resistant to treatment, and cataract are the commonest ocular problems, whilst systemic side effects include hypertension, diabetes, osteoporosis and vertebral collapse, and may become the limiting factors in disease management.

The incidence of steroid-related complications can now be reduced with combination chemotherapy using more selective immunosuppressive drugs, such as cyclosporin $\mathrm{A}$, which allows the steroid dose to be lowered whilst inflammatory control is maintained. Cyclosporin A, an inhibitor of $\mathrm{T}$ cell function, is introduced at a dose of $5 \mathrm{mg} / \mathrm{kg}$ per day, and in many cases this will allow satisfactory reduction in the steroid dose. The blood pressure and serum creatinine are closely monitored, but at this level, in combination with steroids, any rise in serum creatinine that does occur is usually reversible on reduction of the dose of cyclosporin. ${ }^{15}$ The alternative to cyclosporin is azathioprine, usually introduced at a dose of $50 \mathrm{mg}$ three times a day; the blood count must be regularly checked. In some resistant cases, triple combination therapy with steroids, cyclosporin and azathioprine may be required. Chlorambucil has also been reported to have been used with success in sympathetic ophthalmia, although, because of the risk of sterility, its use is limited to the older age group. ${ }^{16}$

Whatever therapy is employed it must be remembered that relapse can occur at any time and therefore close review, with a readiness to increase or reintroduce therapy, is necessary.

Before the use of systemic immunosuppression, sympathetic ophthalmia tended to run a progressively downward course. Today, however, it should no longer be regarded as a blinding disease. The diagnosis is made clinically, Dalen-Fuchs nodules are not a prerequisite for diagnosis, and histological proof is not required. Certainly, injured eyes which have potential vision should not be removed in an attempt to prevent or lessen sympathetic inflammation, or to provide confirmatory pathology. A high index of suspicion must be maintained whenever inflammation occurs in the fellow eye of an eye that has suffered penetrating trauma or intraocular surgery, as delayed diagnosis and treatment may have disastrous, but potentially preventable, consequences.

Key words: Autoimmunity, Azathioprine, Cyclosporin, Immunosuppression, Ocular inflammation, Steroids.

\section{REFERENCES}

1. Fuchs E: Ueber sympathisierende Entzundung (nebst Bemerkungen ueber serose traumatische Iritis). Albrecht $v$ Graefe's Arch Ophthalmol 1905, 61: 365-456.

2. Liddy N, Stuart J: Sympathetic ophthalmia in Canada. Can J Ophthalmol 1992, 7: 157-9.

3. Gass JD: Sympathetic ophthalmia following vitrectomy. Am J Ophthalmol 1982, 93: 552-8.

4. Lubin JR, Albert DM, Weinstein M: Sixty-five years of sympathetic ophthalmia: a clinicopathological review of 105 cases (1913-1978). Ophthalmology 1980, 87: 109-21.

5. Green WR: Uveal tract. In: Spencer WH, editor. Ophthalmic pathology: an atlas and textbook, vol III, 3rd ed. Philadelphia: WB Saunders, 1986.

6. Irvine R: Sympathetic ophthalmia: a clinical review of 63 cases. Arch Ophthalmol 1940, 24: 149-67.

7. Winter FC: Sympathetic uveitis: a clinical and pathological study of the visual result. Am J Ophthalmol 1955, 39: 340-7.

8. Reynard M, Shulman IA, Azen SP, et al.: Histocompatibility antigens in sympathetic ophthalmia. Am J Ophthalmol 1983, 95: 216-21.

9. Jakobiec FA, Marboe CC, Knowles DM III, et al.: Human sympathetic ophthalmia: an analysis of the inflammatory infiltrate by hybridoma-monoclonal antibodies. Ophthalmology 1983, 90: 76-95.

10. Elschnig A: Studien zur sympathetischen ophthalmie. III. Albrecht v Graefe's Arch Klin Exp Ophthalmol 1981, 78: 549-85.

11. Rao NA, Wong VG: Aetiology of sympathetic ophthalmia. Trans Ophthalmol Soc UK 1981, 101: 357-60.

12. Albert DM, Diaz-Rohena R: A historical review of sympathetic ophthalmia and its epidemiology. Surv Ophthalmol 1989, 34: 1-14.

13. Joy HH: Sympathetic ophthalmia: the history of its pathogenic studies. Am J Ophthalmol 1953, 36: 1100-20.

14. Makley TA Jr, Azar A: Sympathetic ophthalmia. Arch Ophthalmol 1978, 96: 257-62.

15. Towler HMA, Whiting PH, Forrester JV: Combination lowdose cyclosporin $\mathrm{A}$ and steroid therapy in chronic intraocular inflammation. Eye 1990, 4: 514-20.

16. Jennings T, Tessler HH: Twenty cases of sympathetic ophthalmia. Br J Ophthalmol 1989, 73: 140-5. 